[Chem. Pharm. Bull.

35( 3 )1228-1233(1987)

\title{
Effect of Cholesterol on Liposome Stability to Ultrasonic Disintegration and Sodium Cholate Solubilization
}

\author{
Toshihisa Yotsuyanagi, * Hotaka Hashimoto, \\ Motokazu Iwata and Ken IKeDa \\ Faculty of Pharmaceutical Sciences, Nagoya City University, \\ Mizuho-ku, Nagoya 467, Japan
}

(Received October 2, 1986)

\begin{abstract}
Liposome disintegration by either ultrasonic vibration or sodium cholate solubilization was investigated as a function of cholesterol $(\mathrm{CH})$ content in the egg phosphatidylcholine (PC) liposome membrane. Turbidity changes were used as an indication of the membrane stability to these stresses. First-order disintegration constants $\left(k_{\mathrm{u}}\right.$ for ultrasonic stress and $k_{\mathrm{s}}$ for sodium cholate solubilization) were calculated to evaluate the membrane stability. A plot of $k_{\mathrm{u}}$ against membrane $\mathrm{CH}$ content gave a sigmoidal curve on which inflection points occurred at about $15 \mathrm{~mol} \%$ and $33 \mathrm{~mol} \%$ $\mathrm{CH}$. In contrast, the plot of $k_{\mathrm{s}}$ against membrane $\mathrm{CH}$ content gave a biphasic curve with only one inflection point at about $17 \mathrm{~mol} \% \mathrm{CH}$. These results can be explained in terms of the molecular packing model of phospholipids and CH proposed by Presti et al. (Biochemistry, 21, 3831 (1982)). Disintegration by ultrasonic stress was little affected by the accumulation of $\mathrm{CH}$-rich domains up to $15 \mathrm{~mol} \% \mathrm{CH}$, but disintegration by sodium cholate was abruptly suppressed in the same $\mathrm{CH}$. content range. These results indicate that the mechanisms of disintegration of $\mathrm{CH}$-rich domains and interfacial boundary phospholipid are entirely different between the two stresses.

In the liposome disintegration by sodium cholate, it was suggested that the penetrationsaturation step of the surfactant molecule into the bilayer is rate-determining for pure PC liposomes, while the lamellar-micellar transition step is rate-determining for $\mathrm{CH}$-rich liposomes.
\end{abstract}

Keywords-liposome stability; cholesterol; egg phosphatidylcholine liposome; ultrasonic vibration; sodium cholate; turbidity change; disintegration mechanism

The physical integrity of liposomes is affected by a variety of external stresses such as ultrasonic vibration, surfactants and freezing. ${ }^{1-6)}$ Ultrasonic vibration, used for the size reduction of vesicles, transforms large multilamellar liposomes into small vesicles. An explanation of this rearrangement process has been given. ${ }^{7)}$ The destruction of liposomal structures by bile salt surfactants may be one of the important factors influencing drug carrier activity after oral administration. ${ }^{3,4)}$

The ability of liposomes to resist degradation by externally applied stresses depends on lipid composition, in particular cholesterol $(\mathrm{CH})$, and also vesicle size and surface charge, ${ }^{8}$ We studied the disintegration of phosphatidylcholine (PC)/CH liposomes caused by ultrasonic vibration and sodium cholate solubilization. The time course of vesicle disintegration was followed by measuring the turbidity changes. Disintegration rate constants obtained from turbidity measurement were used to evaluate the stability of liposomes to these externally applied forces.

\section{Experimental}

Materials_- PC was extracted from egg yolk and purified by column chromatography on silicic acid (Mallinckrodt, St. Louis). ${ }^{9 y}$ Sodium cholate was purchased from Katayama Chem. Co. (Osaka), and recrystallized from acetic acid as cholic acid. $\mathrm{CH}$ and all other chemicals were of reagent grade. 
Preparation of Liposomes-Liposomes were prepared essentially by the method of Bangham et al. ${ }^{10)}$ In short, the required amounts of $\mathrm{PC}$ and $\mathrm{CH}(0-57 \mathrm{~mol} \%)$ were taken in a round-bottomed flask, and dried to give a thin film. Tris buffer ( $\mathrm{pH} 7.4$, I.S. 0.1 with $\mathrm{NaCl}$ ) was added to make a final PC concentration of $1 \times 10^{-3} \mathrm{M}$ based on phosphorus, ${ }^{11)}$ and the mixture was shaken gently then dispersed in a vortex mixer for 3 min.

Disintegration Rate of Liposomes by Ultrasonic Vibration_- Turbidities of the multilamellar liposomes (MLV) prepared above varied significantly even for liposomes of identical lipid composition. Therefore liposome samples were briefly sonicated (probe-type sonifier, model UR 200P, $20 \mathrm{kHz}$, Tomy Seiko Co., Tokyo) for $2.5 \mathrm{~min}$ to obtain reproducible initial turbidities. This preliminary sonication was uniformly applied to all of the liposomes containing $0-57 \mathrm{~mol} \% \mathrm{CH}$. The resulting suspensions were allowed to equilibrate at room temperature for $30 \mathrm{~min}$, which was taken to be $t=0$ for the calculations of disintegration kinetics. Ultrasonic vibration was continuously applied for various periods to each sample, the volume of which was always maintained at $10 \mathrm{ml}$ in a $50 \mathrm{ml}$ glass centrifuge tube under a nitrogen stream at $40^{\circ} \mathrm{C}$. The samples were left standing for $30 \mathrm{~min}$ at room temperature, then turbidity measurements were carried out in a $1 \mathrm{~cm}$ quartz cell at $400 \mathrm{~nm}$, using a Hitachi 124 spectrophotometer.

Disintegration Rate of Liposomes by Sodium Cholate - The disintegration kinetics of liposomes in sodium cholate solution was also followed by measuring the turbidity changes. Turbidity was monitored at $660 \mathrm{~nm}$ by a stopped-flow apparatus (model RA-401, Union Giken, Osaka) equipped with a UV-VIS detector ${ }^{5)}$ Liposomes were first subjected to ultrasonic vibration to give various initial turbidities for the cholate disintegration studies. An equal volume of the liposome suspension containing $3 \times 10^{-4} \mathrm{M}$ phosphorus was quickly mixed with a $0.02 \mathrm{M}$ sodium cholate solution (Tris buffer, pH 7.4, I.S. $0.1,25^{\circ} \mathrm{C}$ ).

The concentrations of lipid and surfactant are diluted to half in the cell of the apparatus. Accordingly, the initial turbidity was presumed to be half of the original turbidity.

Turbidity reduction by ultrasonic vibration or surfactant solubilization was treated as a pseudo-first-order process, and first-order liposome disintegration rate constants, $k_{\mathrm{u}}$ and $k_{\mathrm{s}}$, respectively, were obtained from the turbidity measurements; $k_{\mathrm{u}}$ was calculated from the initial slope of the plots shown in Fig. 1, and $k_{\mathrm{s}}$ was obtained from the linear plots of turbidity disappearance.

\section{Results and Discussion}

\section{Disintegration Behavior under Ultrasonic Vibration}

The sonication system used for the disintegration of liposomes gave reproducible turbidity changes $( \pm 5 \%$ scattering). Unlike the disintegration of vesicles in surfactant solution, in which the bilayer was assumed to be disintegrated by successive stripping of shells from the vesicles, yielding mixed micelles, ultrasonic vibration merely tears the vesicles into smaller bilayer fragments and ultimately finer vesicles. These small vesicles contribute to the total turbidity of the suspension. Because of this, turbidity reduction at the early stages of disintegration was used to calculate $k_{\mathrm{u}}$ values. The rate constant $\left(k_{\mathrm{u}}\right)$ was assumed to represent membrane stability to ultrasonic stress: the smaller the rate constant, the more resistant the bilayer membrane.

Figure 1 shows the turbidity reductions of $\mathrm{CH}$ containing liposomes. At less than $17 \mathrm{~mol} \% \mathrm{CH}$, linearity was generally poor for long sonication times. As the $\mathrm{CH}$ content increased to more than $33 \mathrm{~mol} \%$, the reduction showed an initial slow stage and a subsequent faster stage. Decreasing the sonication stress by using a bath-type sonicator, instead of a probe, prolonged the initial slow stage significantly. ${ }^{2)}$ The reason for this is not clear.

The $k_{\mathrm{u}}$ values were calculated from the initial slope, and also from the terminal linear portion at $\mathrm{CH}$ contents of more than $33 \mathrm{~mol} \%$. Figure 2 shows a plot of $k_{\mathrm{u}} v s . \mathrm{mol} \% \mathrm{CH}$ in the liposomes. The sigmoidal shape of the $k_{\mathrm{u}} v s . \mathrm{mol} \% \mathrm{CH}$ plot had three distinct regions. These regions are defined by the two inflection points at about $15 \mathrm{~mol} \% \mathrm{CH}$ and about $33 \mathrm{~mol} \% \mathrm{CH}$. From 0 to $15 \mathrm{~mol} \% \mathrm{CH}$, the membrane stability remained almost unchanged (stage I). From $15 \mathrm{~mol} \% \mathrm{CH}$ to $33 \mathrm{~mol} \% \mathrm{CH}$, a rather sharp increase in membrane stability occurred (stage II). Increasing $\mathrm{CH}$ beyond $33 \mathrm{~mol} \%$ caused only a moderate increase in membrane stability (stage III). At stage III there was little difference between the increments of the stability obtained from the initial and terminal slopes of the turbidity reductions (Fig. 1).

Presti et al. ${ }^{12)}$ proposed a model for the molecular packing of $\mathrm{CH}$ and phospholipids in membranes. According to their model, phases are formed as a function of the $\mathrm{CH}$ content in 


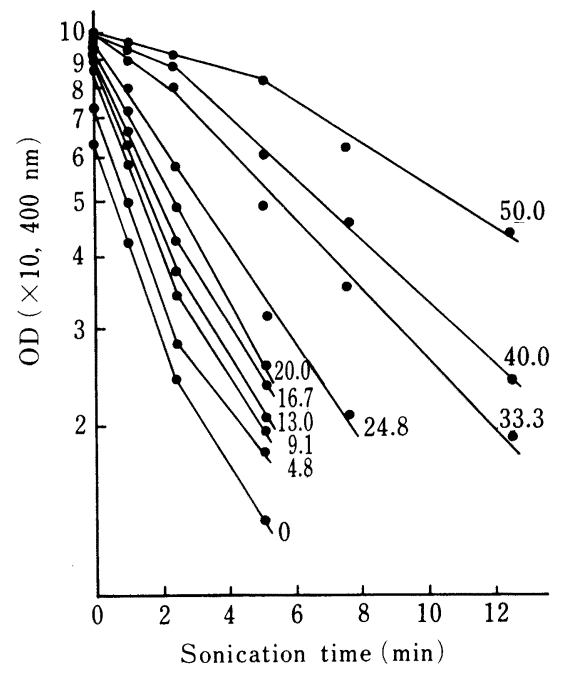

Fig. 1. First-Order Plots of the Turbidity Change due to Liposome Disintegration by Ultrasonic Vibration

The PC concentration is constant at $1 \times 10^{-3} \mathrm{M}$. Numbers indicate $\mathrm{CH}$ content $\left(\mathrm{mol}_{\%} \%\right.$ ). All liposome suspensions were subjected to a prior sonication of 2.5 min. Points are the mean values of $3-5$ determinations and were reproducible better than $\pm 5 \%$.

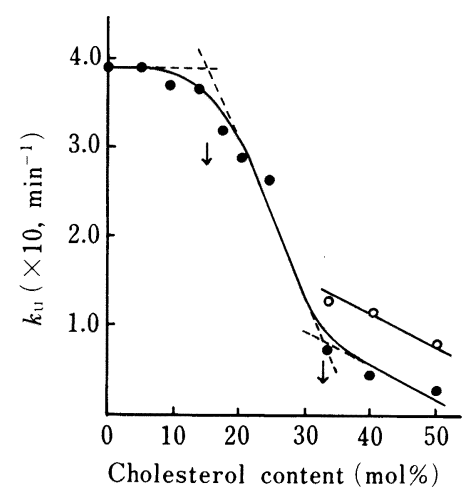

Fig. 2. The Effect of $\mathrm{CH}$ Content on the Disintegration Constant $\left(k_{\mathrm{u}}\right)$ of Liposomes by Ultrasonic Vibration

\footnotetext{
$k_{u}$ was calculated from the initial slope ( was also obtained from the subsequent linear portion for liposomes containing 33.3, 40.0 and $50.0 \mathrm{~mol}^{\circ} \%$ $\mathrm{CH}(\mathrm{O}-\mathrm{O})$. PC concentration, $1 \times 10^{-3} \mathrm{M}$. $\downarrow$ shows an inflection point at which the membrane stability anomalously changes.
}

the bilayer: under $20 \mathrm{~mol}^{\circ} \% \mathrm{CH}, \mathrm{CH}$-rich domains are formed and surrounded by a continuous phospholipid domain or phase. At about $20 \mathrm{~mol} \% \mathrm{CH}$, the continuous PC domain disappears, but interfacial boundary phospholipid remains, separating the $\mathrm{CH}$-rich domains. Further increasing the membrane $\mathrm{CH}$ causes the fraction of interfacial boundary phospholipid to gradually diminish and become zero at $c a .33 .3 \mathrm{~mol} \%$. Uncomplexed phospholipid molecules finally disappear at $50 \mathrm{~mol} \% \mathrm{CH}$.

Based on this model, the relationship between $k_{\mathrm{u}}$ and $\mathrm{mol} \% \mathrm{CH}$ can be interpreted as follows. The membrane stability, i.e. $k_{\mathrm{u}}$, changed very little in stage $\mathrm{I}$, where $\mathrm{CH}$-rich domains are present but do not occupy a large fraction of the membrane. The inflection point observed at $15 \mathrm{~mol}_{0} \% \mathrm{CH}$, which probably corresponds to the first critical ratio in Presti's model, reflects a critical ratio under which the contribution of the $\mathrm{CH}$-rich domains produced in the continuous PC phase to the stability is still minor in the egg PC liposomes. In stage II, the stability increased dramatically with increasing $\mathrm{CH}$ content. This is a reasonable reflection of an accumulation of the $\mathrm{CH}$-rich domains, in other words, a decrease of the interfacial boundary phospholipid. The second inflection point observed at $33 \mathrm{~mol} \% \mathrm{CH}$ coincides with the second critical point $(33.3 \mathrm{~mol} \% \mathrm{CH})$ in the model, at which the bilayer contains only $\mathrm{CH}$ rich domains. Further incorporation $(>33 \mathrm{~mol} \%$ ) of the sterol into liposomes results in a mild increase in membrane stability (stage III) where the $\mathrm{CH}$-rich domains are transformed to the $1: 1$ hydrogen-bonded complexes up to about $50 \mathrm{~mol} \% \mathrm{CH}$. Membranes containing $50 \mathrm{~mol} \%$ $\mathrm{CH}$ are stabilized about four-fold compared to $\mathrm{CH}$ free membranes. This also suggests strong interactions among resulting $\mathrm{PC} / \mathrm{CH} 1: 1$ complexes. It is therefore reasonable to consider that tearing of bilayers by sonication occurs mainly in the free phospholipid domain.

The ultrasonic velocity has been considered to be a good measure of the mechanical properties of membranes. ${ }^{13,14)}$ In the liquid-crystalline state of dipalmitoylphosphatidylcholine (DPPC) liposomes, the limiting value of ultrasonic velocity shows an abrupt increase 
in the region of 15 to $30 \mathrm{~mol}_{\mathrm{o}}^{\mathrm{O}} \mathrm{CH}$. The $k_{\mathrm{u}}$ values also start changing abruptly near $15 \mathrm{~mol} \%$ $\mathrm{CH}$. When egg PC liposomes are used instead of DPPC liposomes, it seems that the stabilizing effect of $\mathrm{CH}$ against ultrasonic vibration is not sensitive to the fatty acid composition of phospolipids but rather to the disappearance of interfacial boundary phospholipid. It is noteworthy that a macroscopic mechanical property represented by the ultrasonic disintegration rate constant $\left(k_{\mathrm{u}}\right)$ is consistent with Presti's model.

\section{Disintegration Behavior in Sodium Cholate}

A mechanistic sequence for the disintegration of liposomes by surfactants has been proposed, in which the mode of action of sodium cholate was classified into type $\mathrm{B} .{ }^{15}$ ) We reported previously that the turbidity disappearance of pure PC liposomes clearly followed first-order kinetics despite the presence of polydispersed vesicles in terms of size. ${ }^{5}$ An explanation was given for the linearity of the first-order plots by introducing the average size factor $(\bar{\phi})$. The reduction rate of the total turbidity $\left(T_{\text {tot }}\right)$ is expressed by

$$
-\frac{\mathrm{d} T_{\mathrm{tot}}}{\mathrm{d} t}=\bar{k} \sum_{i=j}^{m} \frac{A_{i}-A_{i-1}}{A_{i}} A_{i} n_{i} \quad(m>j)
$$

where $\bar{k}$ is the size-independent disintegration rate constant, $A_{\mathrm{i}}$ is the scattering coefficient of the $i$-th vesicle, $n_{\mathrm{i}}$ is the number of the $i$-th vesicle, and $m$ and $i$ denote the size classes of the largest and the $j$-th vesicles sensitive to the turbidity changes at a certain wavelength. The absorbance $(T=A n)$ of a dispersed system containing monodispersed particles is given by ${ }^{16,17)}$

$$
\begin{aligned}
& T=\frac{\ln \left(I_{0} / I\right)}{L}=K \pi a^{2} n \\
& K=K_{0}\left(\frac{a}{\lambda}\right)^{p}
\end{aligned}
$$

where $I_{0}$ and $I$ are the intensities of the incident and transmitted light, respectively. $a$ is the particle radius, $L$ is the length of scattering path, $K$ and $K_{0}$ are the total scattering coefficient and the size-independent component of the scattering coefficient, respectively, and $p$ is the exponent of the wave-length $(\hat{i})$. The term $\left(A_{i}-A_{i-1}\right) / A_{i}$ in Eq. 1 can be therefore represented based on Eqs. 2, 3 and $T=A n$ as

$$
\phi_{i}=\frac{A_{i}-A_{i-1}}{A_{i}}=\frac{a_{i}^{p+2}-a_{i-1}^{p+2}}{a_{i}^{p+2}}
$$

If $\phi_{i}$, which is intrinsically size-dependent, is defined as an average size factor $(\phi)$, Eq. 1 can be represented as a first-order kinetic equation with regard to $T_{\text {tot }}$, i.e.

$$
\begin{aligned}
-\frac{\mathrm{d} T_{\text {tot }}}{\mathrm{d} t} & =\bar{k} \bar{\phi} \sum_{i=j}^{m} A_{i} n_{i} \quad(m>j) \\
& =\bar{k}_{\mathrm{a}} T_{\text {tot }}
\end{aligned}
$$

Accordingly, the experimentally obtained first-order disintegration rate constant $\left(\bar{k}_{\mathrm{a}}\right)$ contains an average size factor $\bar{\phi}$, which may be useful to characterize the disintegration behaviors of dispersed systems with different size distributions.

The turbidity changes of liposomes containing $0-57 \mathrm{~mol} \% \mathrm{CH}$ followed first-order kinetics. Figure 3 shows the pseudo-first-order rate constant $\left(k_{\mathrm{s}}\right)$ obtained as a function of the initial turbidity and $\mathrm{CH}$ content at the constant sodium-cholate concentration of $0.01 \mathrm{M}$. The egg-PC/bile salt molar ratio was maintained at 0.1 throughout, i.e. excess of bile salt. The $k_{\mathrm{s}}$ value depended on the initial turbidities, which reflect different size distributions of vesicles, even when the composition of $\mathrm{PC}$ and $\mathrm{CH}$ was fixed, and the curves appeared to be generally biphasic. Values of $k_{\mathrm{s}}$ for PC liposomes ( $\mathrm{CH}$ free) were most sensitive to the initial turbidity. 


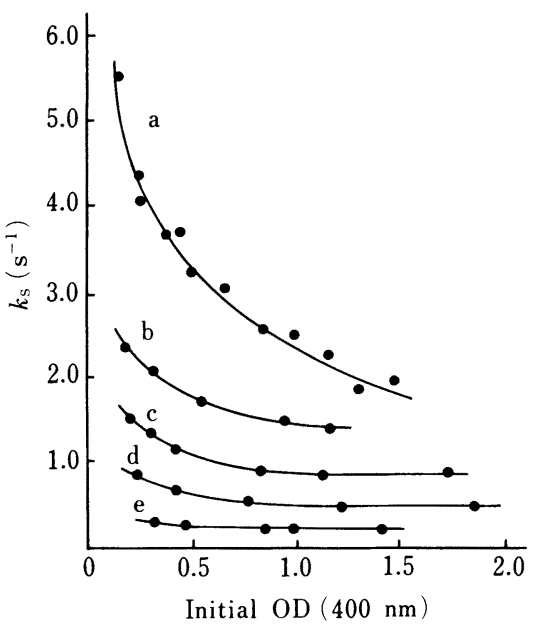

Fig. 3. Effects of Initial Turbidity and $\mathrm{CH}$ Content on the Disintegration Constant $\left(k_{\mathrm{s}}\right)$ in Sodium Cholate Solution

The concentrations of PC and sodium cholate were always maintained at $3 \times 10^{-3}$ and $2.0 \times 10^{-2} \mathrm{M}$, respectively. $\mathrm{CH}$ content $\left(\mathrm{mol}^{\circ} \%\right.$ ): 0 (a), 9.2 (b), 14.0 (c), 29.6 (d) and 57.1 (e). The temperature was $25^{\circ} \mathrm{C}$.

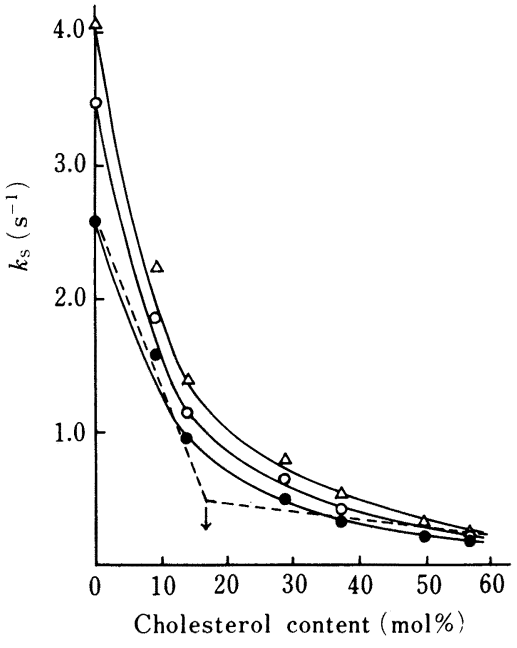

Fig. 4. The Effect of $\mathrm{CH}$ Content on the Disintegration Constant $\left(k_{\mathrm{s}}\right)$ at Different Initial Turbidities

Initial turbidity: $1.0(\bullet), 0.5(\bigcirc)$ and $0.25(\triangle) . \downarrow$ shows an inflection point at which the membrane stability anomalously changes.

Incorporating $\mathrm{CH}$ into the membrane decreased the biphasic nature of the curve (Fig. 3); at about $50 \mathrm{~mol} \% \mathrm{CH}, k_{\mathrm{s}}$ was little affected by the initial turbidity.

In connection with the theory described earlier, the dependency of $k_{\mathrm{s}}$ on the initial turbidity reflects the difference of the size factor; the relationship $k_{\mathrm{s}}=\overrightarrow{k_{\mathrm{a}}}=\bar{k} \bar{\phi}$ holds because $\bar{k}$ is assumed to be a size-independent disintegration constant for a single bilayer. However, as the $\mathrm{CH}$-rich domains were accumulated, the dependency of $k_{\mathrm{s}}$ on the initial turbidity gradually diminished, suggesting that the size factor $\bar{\phi}$ apparently becomes rather constant despite the fact that various initial turbidities were used. These results (Fig. 3) raise the question of why $k_{\mathrm{s}}=\bar{k} \bar{\phi}$ does not always hold for $\mathrm{PC} / \mathrm{CH}$ membranes as the $\mathrm{CH}$ content increases, because we previously estimated $\phi_{i}$ to increase 2.5 times as the vesicle size decreased by half in radius in model calculations ${ }^{5)}$ and such changes of $\phi_{i}$ should be intrinsically applicable irrespective of $\mathrm{CH}$ content. Therefore, the physical meaning of the parameter $\bar{k}$ involved in $k_{\mathrm{s}}$ may differ between the pure $\mathrm{PC}$ liposomes and the $\mathrm{CH}$-rich liposomes. This discrepancy could be accounted for by a stepwise disintegration mechanism of the bilayer. Step I is the penetration-saturation step of bile salt molecules followed by step II, the lamellarmicellar phase transition step.

As shown in Fig. 4, the relationship between $\mathrm{CH}$ content and $k_{\mathrm{s}}$ was biphasic when the initial turbidity was maintained at $1.0 \mathrm{O} \mathrm{D}$. The same tendency was also seen with other initial turbidities $(0.25$ and 0.5$)$. Accumulation of $\mathrm{CH}$-rich domains in the membrane is responsible for the abruptly increasing stability up to about $17 \mathrm{~mol} \%$, and as interfacial boundary phospholipid appears, the membrane shows relatively minor increments of the stability. It was also noted that the $k_{\mathrm{s}}$ dependency showed an inflection point at about $17 \mathrm{~mol} \%$ irrespective of the initial turbidity. It seems likely that with $\mathrm{CH}$-rich liposomes (e.g. 1:1 molar ratio) the amount of the surfactant molecule that penetrates into the bilayer is limited and the transition rate is slowed.

Accordingly, the larger the bilayer in radius, the slower the integration rate. If the $\bar{k}$ reflects the lamellar-micellar phase transition rate for the $\mathrm{CH}$-rich liposomes, it seems 
probable that $k_{\mathrm{s}}=\bar{k} \bar{\phi} \simeq$ constant in consequence of the mutual compensation of the changes in the two parameters, $\bar{k}$ and $\bar{\phi}$. With $\mathrm{CH}$-free liposomes, the penetration-saturation step is rate-determining and $\bar{k}$ is a size-independent parameter; it was assumed previously that the dependency of $k_{\mathrm{s}}$ on the initial turbidity is due to changes of $\bar{\phi}$. Furthermore, the size of the mixed micelles decreases readily. Although the consistency of the theory and results should be further examined, we assume that the disintegration rate of the pure PC liposomes is mainly governed by the former step and with increasing $\mathrm{CH}$ content in the membrane the latter step becomes predominant.

In conclusion, there is a marked difference between the liposome stabilities to ultrasonic vibration (physical stress) and the disintegrating action of cholic acid (chemical stress) as a function of the $\mathrm{CH}$ content. The role of $\mathrm{CH}$ incorporated in egg PC liposomes is often said to be to make the bilayer membrane "more solid-like," but the amounts of $\mathrm{CH}$ used are not always rationalized in the literature. The observed variation of the membrane stability may provide useful criteria for compounding $\mathrm{CH}$ in liposomes and in assessing their response to external stresses.

Acknowledgements This study was supported in part by a Grant-in-Aid for Scientific Research (No. 59570925, No. 60571023) from the Ministry of Education, Science and Culture, Japan.

\section{References}

1) D. Chapman, D. J. Fluck, S. A. Penkett and G. G. Shipley, Biochim. Biophys. Acta, 163, 255 (1968).

2) T. Yotsuyanagi, M. Nonomura and K. Ikeda, J. Parenteral Sci. Technol., 35, 271 (1981).

3) M. H. Richards and C. R. Gardner, Biochim. Biophys. Acta, 543, 508 (1978).

4) R. N. Rowland and J. F. Woodley, Biochim. Biophys. Acta, 620, 400 (1980).

5) T. Yotsuyanagi, J. Mizutani, M. Iwata and K. Ikeda, Biochim. Biophys. Acta, 731, 304 (1983).

6) G. J. Morris and J. J. McGrath, Cryobiology, 18, 390 (1981).

7) W. Helfrich, Phys. Lett., 50A, 115 (1974).

8) J. J. Collins and M. C. Phillips, J. Lipid Res., 23, 291 (1982).

9) A. E. Brandt and W. E. M. Lands, Biochim. Biophys. Acta, 144, 605 (1967).

10) A. D. Bangham, M. M. Standish and J. C. Watkins, J. Mol. Biol., 13, 238 (1965).

11) H. Eibl and W. E. M. Lands, Anal. Biochem., 30, 51 (1969).

12) F. T. Presti, R. J. Pace and S. I. Chan, Biochemistry, 21, 3831 (1982).

13) S. Mitaku, A. Ikegami and A. Sakanishi, Biophys. Chem., 8, 295 (1978).

14) A. Sakanishi, S. Mitaku and A. Ikegami, Biochemistry, 18, 2636 (1979).

15) A. Helenius and K. Simons, Biochim. Biophys. Acta, 415, 29 (1975).

16) G. F. Lothian and F. P. Chappel, J. Appl. Chem., 1, 475 (1951).

17) S. R. Reddy and H. S. Fogler, J. Colloid Interface Sci., 79, 101 (1981). 